Chapter 6

\title{
Effect of Abiotic Stress on Photosystem I-Related Gene Transcription in Photosynthetic Organisms
}

\author{
Dilek Unal Ozakca
}

Additional information is available at the end of the chapter

http://dx.doi.org/10.5772/55350

\section{Introduction}

Photosystem I (PSI) from cyanobacteria and chloroplasts is a multisubunit membrane-protein complex that catalyses electron transfer from reduced plastocyanin in the thylakoid lumen to oxidized ferredoxin in the chloroplast stroma or cyanobacterial cytoplasm [1-4]. PSI is responsible for $\mathrm{NADP}^{+}$reduction and cyclic photophosphorylation and consists of at least 8 polypeptides. Its major components are the P700 chlorophyll a A1 and A2 apoproteins whose molecular weights vary between 60 and $70 \mathrm{kd}$, depending on the species [5]. In this chapter, we discuss recent progress on several topics related to the functions of the PSI complex, like the protein composition of the complex in the plant and algae, the structure and organization of the PSI subunits and the regulation of photosystem I-related gene under abiotic stress conditions. Furthermore, PSI seems to be well protected from photoinhibition in vivo in many plant and algae species and many environmental conditions. The physiology and molecular mechanism during short term adaptation to changes under oxidative stress is discussed in functional and structural terms. Finally, such characteristics of PSI photoinhibition with special emphasis on the relationship between two photosystems as well as the protective mechanism of PSI in vivo is reviewed with respect to function of the thylakoid membrane.

\section{Structure of photosystem I}

PSI catalyzes the light-driven electron transfer from the soluble electron carrier plastocyanin on the luminal side of thylakoid membrane, to ferredoxin on the stromal side of thylakoid membrane. In plants, the PSI complex consists of at least 19 protein subunits, approximately 175 chlorophyll molecules, 2 phylloquinones and $3 \mathrm{Fe}_{4} \mathrm{~S}_{4}$ clusters [6]. The crystal structure of 
PSI from Synechococcus elongatus has also been recently determined at a resolution of less than $4 \AA$ which is sufficient for distinguishing 31 transmembrane and 14 parallel helices and the three ironsulfur clusters [7].

The PSI complex of most plants and algae consists of 13 subunits: at least five chloroplastencoded subunits (PsaA, PsaB, PsaC, PsaI and PsaJ) and eight nucleusencoded subunits (PsaD, PsaE, PsaF, PsaG, PsaH, PsaK, PsaL, PsaN) and numerous redox cofactors and antenna chlorophylls [8]. An additional subunit, PsaM, has only been found in cyanobacteria and in the chloroplast genomes of some lower plants and algae. The PSI subunits PsaG, PsaH and PsaN are only found in eukaryotic photosynthetic organisms and are missing in cyanobacteria $[8,9,10]$.

\subsection{Subunits of PSI}

\subsubsection{PsaA and PsaB}

The major subunits of photosystem I, PsaA and $P s a B$, show strong sequence homology [10, 11] and it was suggested that they have been evolved via gene duplication [12]. They are from the central heterodimer holding the reaction center p700 and components of the electron transport chain (ETC), $\mathrm{A}_{\mathrm{o}}(\mathrm{a} \mathrm{CHLa}), \mathrm{A}_{1}$ (phylloquinon) and $\mathrm{F}_{\mathrm{x}}(\mathrm{a}(4 \mathrm{Fe}-4 \mathrm{~S})$ cluster). In addition, the heterodimer coordinates 80 chlorophylls that function as the instric light-harvesting antenna [13]

Previous studies showed that mutants deficient in PsaB are unable to synthesize both PsaB and PsaA whereas mutants affected primarily in PsaA synthesis are still able to produce PsaB $[2,3,11]$. Based on these results it was proposed that PsaB is an anchor protein during PSI assembly, which needs to be synthesized and integrated into the thylakoid membrane before the other PSI subunits are synthesized [12]. In its absence these polypeptides are no longer synthesized and/or are rapidly degraded. Elucidating how PsaB is translated and inserted into the thylakoid membrane is thus important for understanding the initial steps of PSI assembly.

\subsubsection{PsaC, PsaD and PsaE}

The subunits PsaC, PsaD and PsaE do not contain transmembrane $\alpha$-helices $[3,2,14]$. They are located on the stromal side of the complex, forming the stromal hump. They are in close contact to the stromal loop regions of PsaA and PsaB. Subunit PsaC carries the two terminal FeS clusters FA and FB, and is located in the central part of the stromal hump. PsaD forms the part of this hump, which is closest to the trimeric axis [15,2,3]. The C-terminal part of PsaD forms a 'clamp' surrounding PsaC. PsaE is located on the side of the hump, which is distal from the trimer axis[16,17].

The clusters of PsaC are characterised by their distinct electron paramagnetic resonance (EPR) spectra $[18,19]$. PsaC is likely to posses a pseudo- $C_{2}$ symmetry axis that is oriented perpendicular to distance vector connecting the two iron-sulfur clusters, $\mathrm{F}_{\mathrm{A}}$ and $\mathrm{F}_{\mathrm{B}}$. The role of subunit $\mathrm{PsaC}$ in coordination of the two terminal FeS clusters was suggested from the conserved sequence motif CXXCXXCXXXCP which is found twice in the gene of PsaC [3,20]. A homology 
of subunit PsaC to bacterial ferredoxins, also containing two [4Fe-4S] clusters, was proposed from sequence similarity [21]. The structures of PsaC and these ferredoxins, such as that from Peptostreptococcus asacharolyticus [3,21,22], are indeed similar, with pronounced pseudo-twofold symmetry, concerning previous models which related the structure of subunit PsaC to this ferredoxin $[3,2,21]$.

PsaD is a peripheral subunit of photosystem I (PSI1), an integral protein complex in the thylakoid membrane of oxygenic photosynthetic organisms. Biochemical experiments [20, 21] and analyses of the primary structure of PsaD suggest that it does not posses a transmembranal segment and that it faces the stromal side of the thylakoid membrane. The PsaD is a polypeptide of 139-144 amino acids in cyanobacteria, but has an N-terminal extension of several residues in higher plants, yielding a total length of 158-162 residues [20]. Topological studies $[3,2,19,22,23]$ and data from an X-ray structure of PSI at $4 \AA[24,25]$ show that PsaD probably contains an R-helix and is in contact principally with PsaC and PsaE, and also with PsaH and PsaL $[25,26,27]$. The three-dimensional structure of the higher-plant PSI as determined by electron crystallography has been recently reported [27], confirming that the stromal ridge of higher-plant PSI can also be interpreted as being due to the PsaC, -D, and -E subunits. The N-terminal part of the PsaD subunit can be accessed by the proteases, and its C-terminal region is exposed to solvent [23,14]. Comparison of the amino acid sequences of PsaD from several species shows that the C-terminal part is highly conserved, especially in a region containing many basic residues[23].

In spinach, PsaD is synthesized in the cytoplasm as a precursor of $23.2 \mathrm{kDa}[2,23,21]$ that is processed to produce the mature 17.9-kDa PasD. In vitro assembly assays indicated that both forms of the protein, pre-PsaD and PsaD, can assemble into the thylakoid membranes, specifically into the PSI complex $[2,14,22,24,25,26]$

PsaD is known to interact strongly with ferredoxin. Chemical cross-linking of PSI and ferredoxin consistently yield a product consisting of PSI-D and ferredoxin [14, 23, 25], and recently the interaction has been shown even with isolated PSI-D and ferredoxin [47]. These observations clearly point to an important function of PSI-D in docking of ferredoxin in both eukaryotes and cyanobacteria. The position of ferredoxin in these crosslinked complexes was also identified by electron microscopy [28]. The same docking site was also found for flavodoxin [29] and is in agreement with a docking site proposed from the structural model of PS I at $6 \AA$ A resolution. Subunit PsaD is essential for electron transfer to ferredoxin [28].

PsaE is like PsaD a hydrophilic subunit exposed to the stroma. PsaE is encoded in the nucleus and the mature protein is about $11 \mathrm{kDa}$. Just like PsaD, the mature PsaE in plants has an extended $\mathrm{N}$-terminal region. The extension is variable from 30-40 amino acid residues. As was the case for PsaD, there is no extension in the chloroplast encoded PsaE in Odontella [30], Porphyra [31], and Cyanidium, nor in the cyanelle-encoded PsaE in Cyanophora. Chlamydomonas is peculiar in having PsaE with a short extension of only about ten residues compared to the cyanobacterial PsaE [22].

The structure of subunit PsaE $(8 \mathrm{kDa})$ in solution was determined by $1 \mathrm{H}$ and $15 \mathrm{~N}-\mathrm{NMR}[32$, 33]. The loop connecting L-strands 3 and 4 was found to be flexible in the NMR structure[33]. 
The structure of PsaE in the PSI complex is very similar to the solution structure, with some remarkable deviations in the loop region E-L3L4, which corresponds to the CD loop in the NMR structure. The twist of this loop reported at $4 \AA$ [34] is fully confirmed in the structural model at $2.5 \AA$ resolution. This loop is involved in interactions with PsaA, PsaB and PsaC, suggesting a change of the loop conformation during assembly of the photosystem I complex.

Different functions have been reported for PsaE. PsaE in barley has also been found to be associated with ferredoxin NADP oxidoreductase (FNR) [35]. In cyanobacteria, FNR has a domain linking it to the phycobilisomes [36]. However, recent observations have shown that in spite of this domain, FNR does appear to interact with PsaE [37].

\subsubsection{Other subunits of PSI}

Six small intrinsic membrane protein components of photosystem I have been identified from the gene sequence in S. elongatus [28]: the subunits PsaF (15 kDa), PsaI (4.3 kDa), PsaJ (4.4 kDa), PsaK (8.5 kDa), PsaL (16.6 kDa) and PsaM (3.4 kDa). In the $2.5 \AA$ resolution structure [38, 39, $40]$, a $12^{\text {th }}$ subunit of PS I, PsaX, which contains one transmembrane K-helix, was identified. All of the small membrane integral subunits are located peripherally to the subunits PsaA and PsaB. The main function of the small subunits is the stabilisation of the antenna system and the quaternary structure of photosystem I. The central $\mathrm{Mg}^{2+}$ ions of 10 antenna Chla molecules are axially liganded by amino acid side chains or via water molecules by PsaJ, PsaK, PsaL, PsaM and PsaX. Furthermore, subunits PsaF, PsaI, PsaJ, PsaL, PsaM and to a lesser extent PsaK, are in numerous hydrophobic contacts with the carotenoids[40].

The small subunits can also be divided into two groups according to their location in the complex: PsaL, PsaI and PsaM are located in the region where the adjacent monomers face each other in the trimeric PS I complex, whereas PsaF, PsaJ, PsaK and PsaX are located at the detergent exposed surface of photosystem[40].

PsaF binds the luminal electron donor, plastocyanin [41, 42, 43, 44], and It is essential for providing excitation energy transfer from LHCI to the core complex. Early work showed that PsaF (then called subunit III) was required for electron transfer from Pc to P700 [45, 46]. Subsequently, it was demonstrated that Pc cross-linked to PSI is capable of fast electron transfer to P700 and the cross-linking partner was identified as PsaF.

PsaG and PsaK are two small membrane intrinsic proteins of approximately 10-11 kDa each with two transmembrane $\alpha$-helices connected by a stromal-exposed loop $[47,48]$ and they show a $30 \%$ sequence homology in Arabidopsis [49]. PsaG is unique to plants and green algae. Within the PSI complex PsaK is bound to PsaA and PsaG is bound to PsaB at a roughly symmetry-related position $[49,50]$. PsaK is involved in Lhca3/Lhca2 binding as revealed by knock-out and gene knock-down studies [51, 52, 53].

$\mathrm{PsaH}$ is a $10 \mathrm{kDa}$ protein with one predicted transmembrane helix [54]. The subunit can be chemically cross-linked to PsaD, PsaI and PsaL [50, 54, 55, 56]. Thus, PsaH must be located near the region that constitutes the domain of interaction between monomers in Synechococcus PSI [57]. PSI-H has only been found in plants and green algae. The orientation of PSI-H is not known but based on the positive-inside-rule, the $\mathrm{N}$ terminal region is predicted to be in 
the stroma [58]. Thus, PsaH appears to have about $6 \mathrm{kDa}$ of $\mathrm{N}$ terminal region on the stromal side of the membrane and only about $2 \mathrm{kDa}$ facing the lumen. PSI-H is encoded in the nuclear genome. Apparently, PsaH was a late addition to PSI since it has not been found outside Chlorophyta [54].

PsaN is a small extrinsic subunit of about $10 \mathrm{kDa}$ [54]. PsaN is synthesized with a presequence directing it to the lumen and is the only subunit located exclusively on the lumenal side of PSI [59]. The Psa-G, $-\mathrm{H}$, and $-\mathrm{N}$ fulfill functions in PSI that are unique to eukaryotic PSI. PsaH has been shown to be involved in state 1 -state 2 transitions probably in the interaction with LHCII [54], PsaN is involved in interaction with plastocyanin [41], and PsaG is involved in the stabilization of LHCI and regulation of PSI activity [53]. It is therefore likely that PsaO plays a role in the interaction between the PSI core and other complexes in the thylakoid membrane such as LHCI or LHCII [50, 60]. Alternatively PsaO is involved in the regulation or fine tuning of PSI activity. Together with PsaO, the subunits Psa-G, $-\mathrm{H}$, and $-\mathrm{N}$ are unique to higher plants and algae. Structure of plant PSI at $4.4 \AA$, the structure and position of the Psa-G and $-\mathrm{H}$ subunits within the PSI complex are revealed [49]. However, Psa-N and -O are either not resolved at the current resolution or are lost from the complex during preparation and their structure and exact position in the PSI complex are therefore not known.

PsaJ is a hydrophobic subunit of 4-5 kDa. The protein is chloroplast encoded as is the case also for PsaI, which has a similar size and hydrophobicity [54]. PsaJ is located near PsaF as evidenced by cross-linking [56]. The protein has been thought to be membrane spanning, however, the structural model of cyanobacterial PSI suggest that PsaJ may form an unusual bend helix in the plane of the membrane [60]. In the unicellular green alga Chlamydomonas reinhardtii, PsaJ is required for the stabilization of the PC-binding site [40]. In the absence of PsaJ, a large fraction of photo-oxidized P700 (chl-a dimer of the PSI reaction centre) is not efficiently reduced by PC or cyt (cytochrome) c6, although the PsaF subunit, which forms the actual binding site for both mobile redox carriers, is still present. This has suggested a role of PsaJ in adjusting the conformation of the PC-binding site. These physiological data are circumstantially supported by structural data [49] and cross-linking studies [15] revealing a localization of the J-subunit adjacent to PsaF

Psa-K from spinach may be tightly associated with the PSIA/B heterodimer [61, 62]. However, PsaK from spinach, pea, and barley was depleted from the PSI core by methods used for separation of LHCI from PSI [65, 64]. Treatment of thylakoids with proteases resulted in degradation of PsaK, indicating that part of the PSI-K polypeptide is exposed on the stromal side of the thylakoid membrane. It has therefore been proposed that the membrane-spanning PsaK subunit is located near the rim of the PSI complex between the PSI and LHCI and is thus easily lost upon detergent treatment [51].

There is significant sequence similarity between PsaG and PsaK from eukaryotes [64]. A computer comparison of PsaG and PsaK from Arabidopsis displays approximately $30 \%$ amino acid identity. In fact, the cyanobacterial PsaK is equally similar to plant PSI-G and PsaK. However, there is no evidence that cyanobacterial PSI contains more than one copy of PsaK. In the genome sequence of Synechocystis PCC 6803 two open reading frames have been assigned as potential $p s a K$ genes $[51,65]$. The deduced primary sequences of the two open reading frames 
show only $42 \%$ overall identity. The role of one of the $p s a K$ genes, which encoded a peptide with an amino terminus corresponding to that of the PsaK peptide purified from PSI, has recently been analyzed, and it was shown that the gene product was dispensable for growth, photosynthesis, and the formation of PSI trimers in Synechocystis [65]. The role of the other potential psaK gene remains unknown. However, this gene does not encode a protein with higher similarity to PsaG.

\subsection{Light harvesting complex I}

X-ray crystallography of the PSI core from cyanobacteria $[22,25,50,55]$ as well as modeling studies indicates strong interpigment interactions and unique protein environment as a source for the low energy shifts in absorption of PSI. Biochemical and spectroscopic studies of Light Harvesting Complex I (LHCI) suggest that in the PSI-LHCI super complexes the peripheral antenna and the PSI core antenna have structurally and spectrally distinct pools of red pigments [22]. As in the PSI core antenna, excitonically coupled dimers or trimers of Chl $a$ or $\mathrm{Chl} b$ in the LHCI were suggested to form a pool of red pigments in the LHCI $[2,66,67]$. Recent experiments with antisense inhibited Arabidopsis plants in vivo [68], and reconstitution of the polypeptides in vitro suggests that the presence of low energy pigments is a feature of all four Lhca polypeptides (Lhca1, Lhca2, Lhca3, and Lhca4). Lhca1 seems to possess the less redshifted spectral forms $(684 \mathrm{~nm})[69,70]$, which is in agreement with its close relatedness to a minor light harvesting polypeptide of PSII, CP29. On the contrary, the Lhca4 binds the "reddest" pigments [71]. The presence of the strong fluorescence at $730 \mathrm{~nm}$ in Lhca4 even at room temperature indicates a specific molecular organization of the pigments that efficiently localizes the excitation and could dissipate the excess excitation energy as a nonphotochemical sink when the LHCI antenna is energetically uncoupled from the PSI. Despite a consensus that all the chlorophyll $a / b$ binding proteins (cab) have common evolutionary origin, similar structure and protein sequences [67, 72], there are local structural differences that determine the assembly of Lhca polypeptides around PSI as dimers (Lhca2/Lhca2, Lhca3/Lhca3, and Lhca1/Lhca4) in contrast to the trimer-forming Lhcb polypeptides in LHCII.

\section{Photosytem I antenna}

\subsection{Core antenna}

The antenna of PSI consists of two structurally and functionally parts; the core antenna and preripheral antenna.

The core antenna of PSI contains in total appromeximately 100 chla and $15 \beta$-carotene of which the majority is bound to the PsaA/PsaB dimmer [49]. The chlorophylls have their $\mathrm{Q}_{4}$ absorption maxima around $680 \mathrm{~nm}$. A comparison of absorbtion spectra of PSI, LHCI complexes from wild type A, thaliana and from a mutant lacking the PsaL and PsaH subunits revealed that the about five chlorophylls that are bound to these subunits absorb preferentially at 638 and 667 $\mathrm{nm}$ [73]. 


\subsection{Prephral antenna}

The prepheral antenna of PSI consists of nuclear encoded chlorophyll binding proteins (Lhca) which are transported into the chloroplast and form a light-harvesting complex (LHCI) which increases the light-harvesting capacity of PSI [74].

The protein contacts between the core complex and LHCI appear to be relatively weak, which explains the biochemical sensitivity of the PSI-LHCI supercomplex to detergent attack. It is clear, however, that each of the four light-harvesting proteins fits its specific binding site, because the interface of the core complex formed by subunits PsaG, PsaB, PsaF, PsaJ, PsaA, and PsaK is asymmetric [4, 49]. Lhca1 antenna protein is bound to the core through PsaB and PsaG. Previous studies showed that plants in which $p s a G$ gene expression was suppressed by antisense technology or eliminated by transposon tagging exhibited compromised stability of the antenna and weaker binding of LHCI to the core. Thus, it was proposed that PsaG somehow stabilizes the peripheral antenna. In addition, Lhca3 appears to interact with PsaA. However, the position of newly traced subunit PsaK suggests that it also binds Lhca3. PsaK [39] was proposed to stabilize LHCI organization, and PSI lacking PsaK, isolated from Arabidopsis plants, showed 30-40\% less Lhca3, whereas associations with Lhca1 and Lhca4 were unaffected [75].

\section{Role of the PSI subunits specific to plants and algae}

The extrinsic protein, PsaD, has two reported functions in the PS I complex of cyanobacteria, algae and higher plants. The first function, deduced from in vitro reconstitution experiments, is to stabilize PsaC on the PS I reaction center $[2,23]$. The second function, inferred from crosslinking studies, is to serve as a "docking" protein to facilitate interaction of soluble ferredoxin with the PS I complex [2]. Recent cross-linking experiments have shown that Lys ${ }^{106}$ of PsaD from Synechocystis sp. PCC 6803 can be cross-linked to Glu ${ }^{93}$ in ferredoxin [76]. Therefore these two residues come in physical proximity with each other during at least one stage of electron transfer from PS I to ferredoxin. These results indicate a ferredoxin-docking function of PsaD, but do not illustrate a functional requirement of $\mathrm{PsaD}$ for $\mathrm{NADP}^{+}$photoreduction [77].

While the function of the ten PSI subunits common to plants, algae and cyanobacteria has been studied extensively, the role of the three eukaryotic-specific subunits PsaH, PsaG and PsaN is less well understood. One reason is that these subunits are nucleus-encoded and thus less amenable to genetic manipulation $[2,53]$. However it has recently been possible to generate transgenic Arabidopsis plants lacking PsaH or PsaN by cosuppression or by using an antisense strategy after transformation of the plants with the corresponding cDNAs in the sense or antisense orientation under the control of a constitutive promoter $[41,53,78,79,80]$. PSI complexes lacking the intrinsic PsaH membrane protein contain normal amounts of the other PSI subunits except PsaL which is reduced two-fold [80]. Electron flow through PSI is impaired and the purified PSI complex is highly unstable in the presence of urea. Plants lacking the lumenal PsaN subunit are impaired in the interaction between plastocyanin and photosystem I and the steady-state reduction of $\mathrm{NADP}^{+}$is decreased two-fold [2, 41]. 
The analysis of the PsaF-deficient strain and its suppressor reveals that in the presence of a functional antenna, an intact donor side of PSI is required for protection of $C$. reinhardtii cells from photo-oxidative damage in high light [43]. It is therefore possible that the development of light-harvesting systems for PSI and PSII in eukaryotic organisms demanded an improved donor side of PSI, especially with regard to its functional interaction with the electron donors [81]. This may have led to the evolution of the recognition site within the N-terminal part of PsaF that is essential for fast electron transfer between PSI and its electron donors under high light. It is also possible that plastocyanin replaced cytochrome c6 as electron donor for PSI during evolution because it is slightly more efficient than the heme-containing protein in reducing $\mathrm{P} 700^{+}[2,15,81,82]$ and should therefore provide a beter protection against photooxidative damage.

The function of the PsaF protein $(15 \mathrm{kDa})$ at the lumenal side has been subject to discussion. In intact cells of the green alga Chlamydomonas reinhardtii, PsaF is implicated in the electron transfer from plastocyanin to oxidized P700 by providing a docking site for the electron donor: psaFÿ mutants of this organism had a dramatically reduced electron transfer rate $[15,45,76]$. In contrast, a psaFÿ mutant of the cyanobacterium Synechocystis PCC 6803 exhibited normal electron transfer to P700., implying that PsaF is not essential for the docking of either cytochrome c6 or plastocyanin to PSI [15, 76, 82]. While PSI is extracted as a mixture of trimers and monomers from thylakoid membranes of wild-type cyanobacteria, PSI from mutants that lack the PsaL protein $(16 \mathrm{kDa})$ exists exclusively as a monomer after membrane solubilization [73]. In addition, proteolysis studies have shown PsaL to be located about the 3-fold axis of the trimer, thus holding it together [5, 15]. Little is known about the function of the four other membrane intrinsic subunits (PsaI, -J, -K and -M) that have molecular masses ranging from 3 to $8 \mathrm{kDa}$ [63].

Comparison of deduced primary sequences indicates that the PsaL subunits contain a greater diversity than seen in other subunits $[15,54]$. Function of PsaL in the formation of PS I trimers was revealed by the inactivation of the psaL gene in Synechocystis sp. PCC 6803 [3, 54, 176]. The requirement of PsaL for the trimer formation was later conformed in a PsaL-deficient mutant from Synechococcus sp. PCC 7002 [76, 83]. Recent studies showed that the C-terminus of PsaL is embedded inside the monomeric PS I complex and is involved in trimer formation. Another role of PsaL is in binding to some antenna chlorophyll a molecules.

\section{Gene of photosystem I}

Photosystem I (PSI) is a multiprotein complex in the thylakoid membrane of chloroplasts, providing an interesting system for studying the nucleo-choloroplast relationship in plants.. The core subunits of the PSI reaction centre are still encoded by the chloroplast genome, whereas the genes for the peripheral subunits are located in the nucleus in green algae and land plants. In this study, we dissected the promoter architecture of a nuclear-encoded PSI gene in tobacco, and investigated whether the characteristics found in this promoter are shared by those of the other photosynthesis nuclear genes. 
Sequencing of these proteins and/or their corresponding genes have registered two genes, $p s a A$ and $p s a B$, for the P700-carrying proteins [84], 6 genes, $p s a C$ to $p s a H$, for the core subunits and at least three types of $c a b$ genes for the LHCI subunits [87]. In green plants, $p s a A, p s a B$ and $p s a C$ are encoded by chloroplast genome while the others are encoded by nuclear genome. Sequence comparison indicates that the two P700-carrying proteins and most of the core subunits are more or less conserved between cyanobacteria and green plants, although LHCI is absent in cyanobacteria. In addition to these components, Ikeuchi et al. [88] found that three new lowmolecular-mass components of $4.8 \mathrm{kDa}, 5.2 \mathrm{kDa}$ and $6.8 \mathrm{kDa}$ in cyanobacterial PSI complex and reported their partial amino acid sequences. Interestingly, the sequence of this cyanobacterial $4.1 \mathrm{kDa}$ component corresponded to ORF42/44 of higher plant chloroplast DNA, although its product had not yet been found in plants. Scheller et al. [22] reported the presence of two small proteins below $4 \mathrm{kDa}$ in higher plant PSI complex. These prompted us to search for the product(s) of ORF42/44 in higher plant PSI complex as well. Here we report the presence of three lowmolecular- mass proteins in spinach and pea PSI complexes and their unambiguous identification by $\mathrm{N}$ terminal sequencing.

The genes for the two subunits, psaA and psaB, are located adjacent to each other in the large single-copy region of circular plastid genome in higher plants [89]. Gene psaB is followed by rps14 encoding the chloroplast ribosomal protein CS14 [90]. The psaA-psaB-rps14 gene cluster was found to co-transcribe into a 5- to 6-kb polycistronic mRNA in spinach [91], tobacco [90], and rice [89]. Cheng et. al. [90] have performed a detailed transcriptional analysis of the promoter of rice psaA-psaB-rps14 operon with deleted mutants in vitro. They showed that two functional promoters denoted as "-175" and "-129" were revealed.

Some of the known signals which are targeted to these genes are light, plastid signal(s) and hormones. Nuclear encoded genes of PSI are effectively activated by white and red light, while blue light is less effective. Essential promoter units which are responsive to light were identified by deletions and mutational analyses. Another set of signals is produced by the plastids reporting the state of the plastids to the nucleus. When plastids were bleached by the addition of norflurazon, transcription of nuclear PSI genes was decreased [92]. The nature of these plastid-derived signals has still to be elucidated, however it appears that the communication between the two organelles is mediated by more than one signal $[93,94]$. The responsive units within the promoter appear to be the same as the light-responsive elements [93]. The third group of signals acting on genes for thylakoid proteins is plant hormones. Kusnetsov et al. [95] suggested that it could be shown that cytokinin stimulated the transcription of AtpC and $\mathrm{Cab}$ genes in the dark. Even the formation of thylakoid membranes could be stimulated by cytokinin. Antagonistic to the cytokinin effect abscisic acid decreased transcription of target genes. In addition to these types of regulation PSI genes were also regulated by daytime. The peaks of transcriptional activities of $P_{s a E}, P_{s} a F, P_{s a G}$ and $P_{s} a H$ were one hour after the onset of light [92]. The oscillation stopped in continuous light. The regulatory interactions leading to this phenomenon are not known at present. However, it became obvious that all of these signaling systems controlling nuclear gene expression can only operate fully when functional plastids are available [92]. Therefore we examined whether the expression of a photosynthesis 
promoter-driven reporter gene construct is also expressed in roots and whether elements can be distinguished which respond differently to externally applied signals.

In the spinach PsaF promoter a CAAT box is located near the transcription start site (at position -178/-174). A similar motif has been found in the light-reactive promoter unit of the PsaD gene in Nicotiana sylvestris [96]. A protein factor binding to such a motif has been isolated from Arabidopsis thaliana and was shown to be regulated by light, cytokinin, and the stage of the plastid [97]. While the CAAT box region appears to be sufficient for regulated expression of $A t p C$, hardly any expression could be detected for a CAAT box containing PsaF fragment lacking the region upstream of $-179[97,98]$. In gel retardation studies binding of a nuclear factor to the adjacent $P$ saF promoter region -220/-179 could be demonstrated [99]. The -220/-179 region was not only shown to be a stimulator of $P_{s a F}$ expression, but also enhances expression in response to different stimuli. It includes a GT-1 binding site (-194/-180) [100]. Results of this study show that in transgenic tobacco roots this promoter region is crucial for the activation by cytokinin, mastoparan and $\mathrm{Ca}^{2+}$. This suggests that phosphoinositides, such as inositol-3phosphate, and a $\mathrm{Ca}^{2+-}$ dependent kinase are involved in regulating transcription factor activity operating on this promoter region.

In higher plants, the function of the nuclear-encoded subunits has been elucidated in recent years using RNAi (RNA interference), antisense techniques and insertional mutagenesis in Arabidopsis thaliana [101]. This work has revealed non-essential functions of most nuclearencoded PSI subunits, with the exception of PsaD, which is essential for PSI accumulation and is involved in the formation of the ferredoxinbinding site [101]. All other knockout mutants displayed much weaker phenotypes, often including a reduced PSI content due to destabilization of the complex and sometimes showing altered antenna binding and exciton transfer to the reaction centre [16].

\section{Photoinhibition of PSI and response to abiotic stress}

Plant chloroplasts include two large pigment-protein complexes, such as photosystem I and photosystem II that are located within thylakoid membranes. The reaction centres of PSI and PSII are formed by chlorophyll a-binding heterodimers, PsaA/PsaB and PsbA/PsbD proteins, respectively. PSI and PSII are both organized into supercomplexes with variable amounts of nuclear-encoded chlorophyll a/b-binding proteins forming light-harvesting antenna complexes around PSI (LHCI) and PSII (LHCII) (Figure 1).

Environmental factors such as temperature, UV-light, irradiance, drought and salinity are known to affect photosynthesis in both cyanobacteria and plants (Figure 1). In cyanobacteria, several studies have been reported on photosynthetic electron transport activities both under salt and high light stress conditions in whole cells as well as thylakoid membranes[102,103]. 


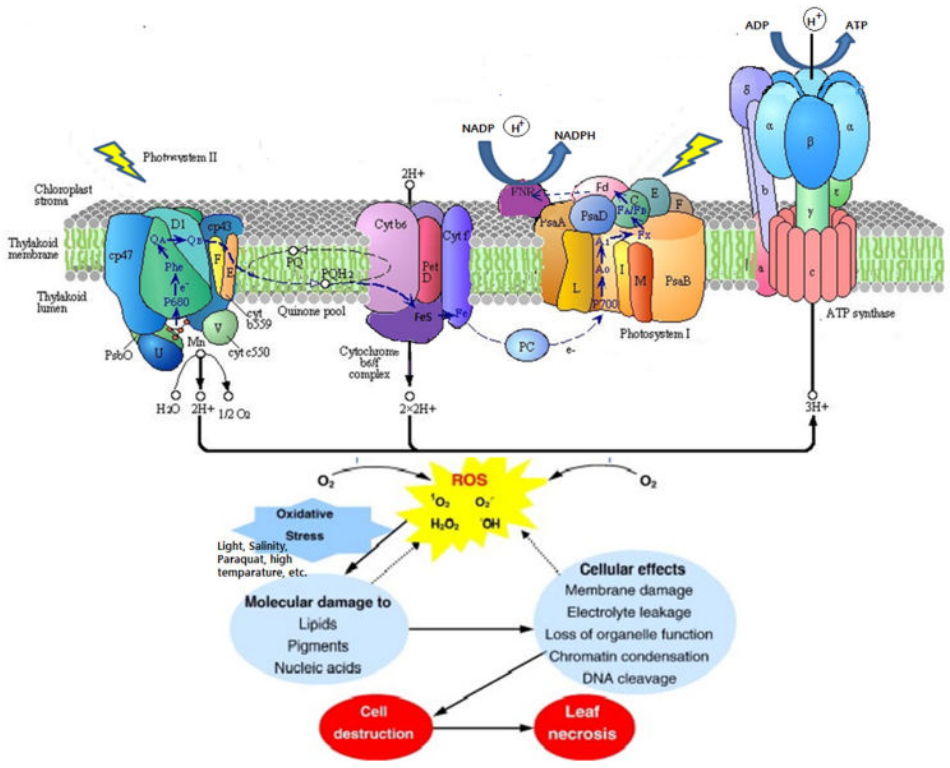

Figure 1. Schematic figure of oxidative stress effects on PSII and PSI

Photoinhibition of Photosystem I (PSI) was first reported by Jones and Kok [104], is the one who originally called them 'photoinhibition' [105]. The subsequent studies revealed that the activity of PSI could be photoinhibited in thylakoid membranes $[106,107]$ as well as in isolated PSI complexes [108, 109]. However, PSI-specific photoinhibition was never observed in intact leaves until 1994 [110]. The selective photoinhibition of PSI was first observed in cucumber leaves treated at chilling temperatures [111]. In contrast PSI is generally believed to be less sensitive to light stress and its photoprotection mechanisms were less investigated. Nevertheless, several recent evidences showed that PSI can also be targeted by photoinhibition, especially under chilling conditions and when the linear electron transport chain is unbalanced $[111,112]$. PSI photoprotection has been suggested to be mainly mediated by oxygen scavenging enzymes (e.g., superoxide dismutase and ascorbate peroxidase) which efficiently detoxify reactive species produced at the reducing side of Photosystem I [113]. A decreased ability of these enzymes to scavenge ROS at low temperatures was proposed to be the reason of the major PSI photo-sensitivity in chilling conditions [111, 112, 114]. As a summary, processes during of PSI photoinhibition as follow;

1. Decreased rate of reducing power utilization by Calvin cycle enzymes (Rubisco) at low temperature;

2. Photoinduced electron transfer from PS2 and reduction of PS1 electron acceptors (FeS centres, Fd, NADP);

3. Cold-induced diminish of oxidative defense system (tAPX, sAPX etc.) capacity; 
4. Recombination of separated charges in PS1 reaction centres between $\mathrm{P}^{-} 00^{+}$and $\mathrm{A}_{0}{ }^{-}$or $\mathrm{A}^{1-}$ and Chl triplet formation;

5. Energy migration from $\mathrm{TChl}$ to $\mathrm{O}^{2}$ and production of singlet oxygen ${ }^{1} \mathrm{O}^{2}$;

6. Superoxide anion radical and $\mathrm{H} 2 \mathrm{O} 2$ production in Mehler reaction;

7. Fenton reaction $\left(\mathrm{OH}^{\bullet}\right.$ formation as result of interaction of $\mathrm{H}_{2} \mathrm{O}_{2}$ with reduced FeS-clusters);

8. Destruction of FeS-clusters by $\mathrm{OH}$;

9. Inactive FeS-clusters induce the conformational changes of PS1 core complex proteins facilitating its access for proteases;

10. Degradation of PsaB and PsaA gene products and release of $45 \mathrm{kDa}$ and 51kDa proteins;

11. Processes (8) and (10) result PS1 photoinhibition

The eventual effect of the abiotic stress on plant growth and crop productivity is a result not only of the extent of the damage but also on the capacity for recovery after the damage has taken place. Although the recovery and repair of PSII after photoinhibition have been a subject of many studies [115, 116, 117], there is very little known about the recovery and repair of PSI. Teicher et. al. [118] showed that PSI recovery is a very slow process, which may take several days even under optimal conditions in field-grown barley. A more recent study showed that PSI damage in cucumber is not even completely reversible [119]

The few previous studies have not shown whether PSI repair is similar to PSII repair where one particular subunit, D1, is specifically remade whereas the rest of the complex is reused [120]. Clearly, the PSI repair process must involve some protein turnover but it is not known whether the breakdown that is observed during photodamage is caused directly by the damage or is part of the recovery process.

Light are highly unpredictable resource for plants and the changes in growth irradiance induce several changes in biochemical and molecular composition of the plant cell. Murchie et al. [121] showed that there are 99-light responsive genes which were down regulated and 130 were upregulated in rice during light treatment. Majority of these genes showed reduced levels of expression in response to high light, whereas stress related genes showed increased level of expression. In order to avoid over-excitation of chlorophyll protein complexes and photooxidation, a regulated degradation of LHC was observed in rice leaves along with a decline in CP-24, PSI genes and a $10 \mathrm{kD}$ PSII gene was also noticed under high light [121].

PS I has long been reported to be less affected than PSII by high light [105]. PSI in isolated thylakoid membranes was inactivated by high light [122]. Since PSI is the terminal electron carrier in the chloroplast, it was identified as a major site producing ROS and shown to be closely associated with ROS-scavenging systems in the chloroplast [123]. The role of ROS inactivating PSI reaction center and degradation of psaA and psaB under high light conditions has been studied [124]. Very recently, Jiao et al [125] demonstrated that high light stress readily photoinhibited PSI, following the loss of $\mathrm{psaC}$ as well as degradation of PSI reaction center proteins (psaA and psaB). The findings suggest that PSI photoinhibition can be a limiting factor in crop productivity under high light. 
Several studies demonstrated that thylakoid memebrane proteins were affected by salt stress. In Synechococcus cells, $\mathrm{NaCl}$ at $0.5 \mathrm{M}$ concentration inactivated both PSII and PSI due to the changes in K/Na [102]. Lu and Voshak [126, 127] have also been demonstrated that salt stress itself has no direct effect on PSII activity in Spirulina plantensis preincubated in the dark, but the same salt stress in combination with Photosynthetically active radiation (PAR) led to block of electron transport between $\mathrm{Q}_{\mathrm{A}}$ and $\mathrm{Q}_{\mathrm{B}}$ (primer and secondary quinine electron acceptors of PSII), and the inhibition of PSII electron transport was proportional to the intensity of light. In addition, it has been revealed that cyclic electron flow around PSI was enhanced during salt stress in cyanobacteria [128,129]. Zhang et al. [103] observed that PSI activity in salt-stressed cells increased. Sudhir et al. [130] also reported that salt stress induced an increase in PSI activity. The increased PSI activity may be due to an increase in the content of P700 reaction centers[131,132]. An increase in PSI activity should increase cyclic electron transport. Several reports have shown that cyclic electron flow increases under salinity stress[130]. Hence, it seems that an increase in PSI activity in salt-adapted cells may protect PSII from excessive excitation energy under salt stress.

A few reports have shown the effects of metals on the activity of photosystem I (PSI) and some of them was controversial. Neelam and Rai [133] reported that cadmium treatment inhibits PSI activity in Microcystis sp. Zhou et al. [134] also suggested that the increase in PSI activity results from the increase of cyclic electron transport around PSI, which increases of ATP synthesis. At the same time, this cyclic electron transport around PSI is suggested to play an important role in the synthesis of more ATP, which would provide more energy to maintain proper defense system within the cell. In addition, Ivanov et al [135] showed that increased PSI mediated cyclic electron transport would be observed in plants which was grown under temperature / light stress conditions. It has been suggested that cyclic electron flow around PSI is required to supply sufficient proton motive force to initiate energy-dependent excitation quenching $\left(\mathrm{q}_{\mathrm{E}}\right)$. Jin et al. [136] showed that cyclic electron flow around PSI plays an important role in the production of $\mathrm{pH}$ gradient across the thylakoid membrane $(\Delta \mathrm{pH})$ that leads to the effective dissipation of excess excitation energy under high temperature conditions. Thus, the increase in PSI activity resulting from the increase of cyclic electron transport around PSI could be one of the adaptive mechanisms to stress conditions [137, 138, 139].

\section{Author details}

\section{Dilek Unal Ozakca}

Address all correspondence to: dilek.unal@bilecik.edu.tr, dilek.unal@mail.ege.edu.tr

Bilecik Seyh Edebali University, Faculty of Science and Art, Department of Molecular Biology and Genetic, Bilecik, Turkey 


\section{References}

[1] Chitnis P. R Photosystem I: Function and Physiology Annu Rev Plant Physiol Plant Mol Biol. 2001; 52:593-626.

[2] Scheller H.V, Jensen P. E, Haldrup. A, Lunde. C, Knoetzel J Role of subunits in eukaryotic Photosystem I. Biochim Biophys Acta 2001;1507: 41-60

[3] Manna P., Chitnis P.R Function and molecular genetics of Photosystem I, in: G.S. Singhal, G. Renger, S.K. Sapory (Eds.)i Concepts in Photobiology:Photosynthesis and Photomorphogenesis, 1998, pp.212-251

[4] Amunts A., Nelson N Fuctional organization of a plant Photosystem I:Evolution of a highly efficient photochemical machine. Plant Physiol. Biochem. 2008; 46:228-237

[5] Jensen P.E, Bassi R, Boekema E.J, Dekker J.P, Jansson S, Leister D, Robinson C, Scheller H.V Structure, function and regulation of plant photosystem I, Biochim. Biophys. Acta 2007;1767:335-352

[6] Ben-Shem A, Frolow F, Nelson N Crystal structure of plant photosystem I, Nature 2003;426:630-635

[7] Klukas O., Schubert WD., Jordan P., Krauss N., Framme P., Witt HT., Seanger W., 1999. Photosystem I, an improved model of the stromal subunits PsaC, PsaD, and PsaE. J. Biol. Chem. 274(11):7351-60.

[8] Ihnatowicz A., Pesaresi P., Varotto C., Richly E., Scheider A., Jahns P., Salamini F., Leister D Mutants of photosystem I subunit D of Arabidopsis thaliana : effects on photosynthesis, photosystem I stability and expression of nuclear genes for chloroplast functions. Plant J. 2004; 37, 839-852

[9] Jensen P. E., Haldrup A., Rosgaard L., Scheller, H. V Molecular dissection of photosystem I in higher plants: topology, structure and function. Physiol. Plant. 2003;119, 313-321

[10] Rochaix J. D, Fischer N, Hippler M Chloroplast site-directed mutagenesis of photosystem I in Chlamydomonas: Electron transfer reactions and light sensitivity Biochimie 82 2000; 635-645.

[11] Oh-oka H, Takahashi Y, Matsubara H Topological considerations of the 9-kDa polypeptide which contains centers A and B, associated with the 14- and 19-kDa polypeptides in the photosystem I complex of spinach. Plant Cell Physiol 1989; 30: 869-875

[12] Vallon O, Bogorad L Topological study of PSI-A and PSI-B, the large subunits of the photosystem-I reaction center. Eur J Biochem 1993; 214: 907-915

[13] Hoj P.B., Svendsen I., Scheller H.V., Moller B.L. Identification of a chloroplast-encoded 9-kDa polypeptide as a $2[4 \mathrm{Fe}-4 \mathrm{~S}]$ protein carrying centers A and B of photosystem I J. Biol. Chem. 1987; 262:12676-12684. 
[14] Xia Z., Broadhurst R.W., Laue E.D., Bryant D.A., Golbeck J.H., Bendall D.S Structure and properties of PsaD in solution. Eur. J. Biochem. 1998; 255:309-316.

[15] Chitnis P.R., Xu Q., Chitnis V.P., Neshushtai R Function and organization of photosystem I polypeptides, Photosynth Res. 1995;44: 23-40

[16] Haldrup A., Simpson D.J, Scheller H.V Down-regulation of the PSI-F subunit of photosystem I in Arabidopsis thaliana. The PSI-F subunit is essential for photoautotrophic growth and antenna function, J. Biol. Chem. 2000;275:31211-31218.

[17] Varotto C., Pesaresi P., Jahns P., Lessnick A., Tizzano M., Schiavon F., Salamini F., Leister D Single and double knockouts of the genes for photosystem I subunits G, K, and $\mathrm{H}$ of Arabidopsis. Effects on photosystem I composition, photosynthetic electron flow, and state transitions, Plant Physiol. 2002;129:616-624.

[18] Allen JF. Redox control of gene expression and the function of chloroplast genomes - an hypothesis. Photosynth. Res. 1993; 36:95-102.

[19] Pfannschmidt T, Nilsson A, Allen JF Photosynthetic control of chloroplast gene expression, Nature 1999;397:625-628.

[20] Klukas O, Schubert W.D, Jordan P, Krauss N, Fromme P, Witt H.T, Saenger W., Photosystem I, an improved model of the stromal PsaC, psaD and psaE. J. Biol. Chem. 1999;274:7351-7360.

[21] Vassillev I.R, Jung Y.S, Yang F, Golbeck J.H PsaC subunit of photosystem I is oriented with iron-sulfur cluster $\mathrm{F}_{\mathrm{B}}$ as the immediate electron donor to ferredoxin and flavodoxin. Biophys. J 1998; 74:2029-2035,

[22] Scheller H. V, Jensen P. E, Haldrup A, Lunde C, Knoetzel J. Role of subunits in eukaryotic photosystem I. Biochim. Biophys. Acta 2001;1507:41-60

[23] Jin P, Sun J, Chitnis P.RStructural features and assembly of the soluble overexpressed psaD subunit of Photosystem I. Biochm. Biophys. Acta 1999; 1410:7-18

[24] Krauß N, Schubert W-D, Klukas O, Fromme P, Witt HT, Saenger W Photosystem I at $4 \AA$ resolution represents the first structural model of a joint photosynthetic reaction and core antenna system. Nat Struct Biol 1996; 3:965-973.

[25] Shubert WD, Klukas O, Krauß N, Saenger W, Framme P, Witt H. T Photosystem I of Synechoccus elongarus at $4 \AA$ resolution:comprehensice structure analysis, J. Mol. Biol. $1997 ; 272: 741-769$

[26] Xu Q, Armbrust T.S, Guikema J.A, Chitnis P.R, Organization of Photosystem I polypeptides: A structural interaction between psaD and psaL subunits. Plant Physiol. 1994;106:1057-1063

[27] Yu J, Smart L.B, Yung Y.S, Golbeck J, McIntosh L, Absence of PsaC subunit allows assembly of photosystem I core but prevents the binding of PsaD and PsaE in Synechocyctis sp. PCC6803. Plant Mol. Biol. 1995; 29:331-342 
[28] Chitnis P.R Photosytem I. Plant Physiol. 1996;111:661-669

[29] Lelong C, Setif P, Laqoutte B, Bottin H, Identification of the amino acids involved in the functional interaction between photosystem I and ferrodoxin from Synechocystis sp. PCC6803 by chemical cross-linking. J. Biol. Chem. 1994; 269:10034-10039

[30] Kowallik K.V, Stoebe B, Schaffran I, Kroth-Pancic P.G, Freier U The chloroplast genome of a chlorophyll a+c containing alga, Odontella sinensis. Plant Mol. Biol. Rep. 1995; 13:336-342

[31] Reith M, Munholland J Complete nucleotide sequence of the Porphyra purpurea chloroplast genome. Plant Mol. Biol. Rep. 1995; 13:333-335

[32] Falzone C.J, Kao Y-H, Zhao J, MacLaughlin K.L, Bryant D.A, Lecomte, J.T.J ${ }^{1} \mathrm{H}$ and ${ }^{15} \mathrm{~N}$ NMR assignments of PsaE, a photosystem I subunit from the cyanobacterium Synechococcus sp. Strain PCC 7002. Biochemistry 1994;33: 6043-6051.

[33] Falzone C.J, Kao Y.H, Zhao J, Bryant D.A, Lecomte J.T Three-dimensional solution structure of PsaE from the Cyanobacterium Synechococcus sp. Strain PCC 7002, a photosystem I protein that shows structural homology with SH3 domains. Biochemistry 1994; 33(20):6052-62

[34] Jordan P, Fromme P, Witt H.T, Klukas O, Saenger W, Krauss N Three-dimensional structure of cyanobacterial photosystem I at $2.5 \AA$ resolution. Nature 2001;411:909-917

[35] Andersen B., Scheller H.V, Møller B.L The PSI-E subunit of photosystem I binds ferredoxini $\mathrm{NADP}^{+}$oxidoreductase. FEBS Lett 1992; 311:169-173

[36] Schluchter WM., Bryant DA. Molecular characterization of ferrodoxin-NADP ${ }^{+}$oxidoreductase in cyanobacteria: cloning and sequence of the petH gene of Synechococcus sp PCC 7002 and studies on the gene product. Biochemistry 1992;31:3092-3102

[37] van Thor J.J, Geerlings T.H, Matthijs H.P, Hellingwerf K.J Kinetic evidence for the PsaE-dependent transient ternary complex photosystem I/Ferrodoxin/Ferrodoxin: $\mathrm{NADP}^{+}$reductase in a cayanobacterium. Biochemistry 1999; 38:12735-12746

[38] Xu Q, Odom W.R, Guikema J.A, Chitnis V.P, Chitnis P.R Targeted deletion of psaJ from the cyanobacterium Syneckocystis sp. PCC 6803 indicates structural interactions between the PsaJ and PsaF subunits of photosystem I. Plant Mo1 Biol 1994; 2624:291-30

[39] Jensen P.E, Gilpin M, Knoetzel J, Scheller H.V The PSI-K subunit of photosystem I is involved in the interaction between light-harvesting complex I and the photosystem I reaction center core, J. Biol. Chem. 2000;275:24701-24708.

[40] Schöttler M.A, Flügel C, Thiele W, Stegemann S, Bock R The plastome-encoded PsaJ subunit is required for efficient Photosystem I excitation, but not for plastocyanin oxidation in tobacco. Biochem. 2007;403:251-250 
[41] Haldrup A, Naver H, Scheller H.V, The interaction between plastocyanin and photosystem I is inefficient in transgenic Arabidopsis plants lacking the PSI-N subunit of photosystem I. Plant J. 1999;17:689-98

[42] Haehnel W, Janse T, Gause K, Klosgen R.B, Stahl B, Michl D, Huvermann B, Karas M, Herrmann R.G Electron transfer from plastocyanin to photosystem I EMBO J. 1994;13:1028-1038.

[43] Farah J, Rappaport F, Choquet Y, Joliot P, Rochaix J.D Isolation of a psaF-deficient mutant of Chlamydomonas reinhardtii: efficient interaction of plastocyanin with the photosystem I reaction center is mediated by the PsaF subunit. EMBO J. 1995;14(20): 4976-84

[44] Hippler M., Drepper F., Farah J, Rochaix J.D Fast electron transfer from cytochrome c6 and plastocyanin to photosystem I of Chlamydomonas reinhardtii requires PsaF. Biochemistry 1997;36:6343-6349

[45] Filieger K, Tyagy A, Sopory S, Csepiö A, Hermann R.G, Oelmüller R A 42 bp fragment of the gene for subunit III of photosystem I (PsaF) is crucial for its activity, Plant J 1993;4:9-17

[46] Oelmüller R, Lübberstedt T, Bolle C, Sopory S, Tyagy A, Csepiö A, Filieger K, Hermann R.G 1992. Promoter architecture of nuclear genes for thylakoid membrane proteins from spinach. In:Murata N (ed) Research in Photosynthesis Vol III. Kluwer Acad Publishers, Dordrecht pp 219-224.

[47] Mant A, Woolhead C.A, Moore M, Henry R, Robinson C Insertion of PsaK into the thylakoid membrane in a "horseshoe" conformation occurs in the absence of signal recognition particle, nucleoside triphosphates or functional Albino3. J. Biol. Chem. 2001;276:36200-36206

[48] Rosgaard L, Zygadlo A, Scheller H.V, Mant A, Jensen P.E Insertion of the plant photosystem I subunit $G$ into the thylakoid membrane in vitro and in vivo studies of wild-type and tagged versions of the protein. FEBS J. 2005; 272:4002-4010

[49] Ben-Shem A, Frolow F, Nelson N Crystal structure of plant photosystem I. Nature 2003; 426:630-635

[50] Jensen P.E, Bassi R, Boekema E.J, Dekker J.P, Jansson S, Leister D, Robinson C, Scheller H.V Structure, function and regulation of plant photosystem I. Biochim. Biophys. Acta 2007;176:335-352

[51] Jensen P.E, Glipin M, Knoetzel J, Scheller H.V The PSI-K subunit of photosystem I is involved in the interaction between light-harvesting complex I and the photosystem I reaction center core. J. Biol Chem 2000;275:24701-24708

[52] Jensen P.E, Rosgaard L, Knoetsel J, Scheller H.V Photosystem I activity is increased in the absence of the PSI-G subunit. J. Biol. Chem. 2002; 277:2798-2803 
[53] Varotto C, Pesaresi P, Jahns P, Leßnick A, Tizzano M, Schiavon F, Salamini F, Leister D Single and double knockouts of the genes for photosystem I subunits $G, K$, and H of Arabidopsis, effects on photosystem I composition, photosynthetic electron flow, and state transitions. Plant Physiol. 2002; 129:616-624

[54] Scheller H.V, Jensen P.E, Haldrup A, Lunde C, Knoetzel J Role of subunits in eukaryotic Biochim Biophys Acta. 2001;1507(1-3):41-60.

[55] Andersen B, Koch B, Scheller H.VStructural and functional analysis of the reducing side of photosystem I. Physiol. Plant. 1992; 84:154-161

[56] Jansson S, Andersen B, Scheller H.V Neraest-neighbor analysis of higher-plant photosystem I holocomplex. Plant Physiol. 1996;112:409-4020

[57] Schubert W.D, Klukas O, Krauss N, Saenger W, Framme P, Witt H.T Photosystem I of Synechococcus elongates at 4 angstrom resolution. Comprehensive structure analysis. J. Mol. Biol. 1997;272:741-769

[58] Vonheijne G Membrane protein structure prediction, hydrophobicity analysis and the positive-inside rule. J. Mol. Biol. 1992; 225:487-494.

[59] Nielsen V.S, Mant A, Knoetzel J, Møller B.L, Robinson C Import of barley photosystem I subunit $\mathrm{N}$ into the thylakoid lumen is mediated by a bipartite presequence lacking an intermediate processing site. Role of the delta $\mathrm{pH}$ in translocation across the thylakoid membrane. J. Biol. Chem. 1994; 269(5):3762-3766

[60] Jensen P. E, Haldrup A, Zhang S, Scheller, H. V The PSI-O subunit of plant photosystem $\mathrm{I}$ is involved in balancing the excitation pressure between the two photosystems. J. Biol. Chem. 2004; 279, 24212-24217

[61] Hoshina S, Sue S, Kunishima N, Kamide K, Wada K, Itoh S Characterzation and Nterminal sequence of a $5 \mathrm{kDa}$ polypeptide in the photosystem I core complex from spinach FEBS Lett 1989; 258:305-308

[62] Wyn R.N, Malkin R The photosystem I $5.5 \mathrm{kDa}$ subunit (the psaK gene product) an intrinsic subunit of the PSI reaction center complex. FEBS Lett 1990; 262:45-48

[63] Ikeuchi M, Hirano A, Hiyama T, Inoue Y Polypeptide composition of higher plant photosystem I complex Identification of psaI, psaj and psaK gene products. FEBS 1990;263(2):274-278

[64] Kjaerulff S, Anderson B, Skovgaard N.V, Møller L.B, Okkels J.S The PSI-K subunit of the photosystem I from barley (Hordeum vulgare L.): Evidence for a gene duplication of an ancestral PSI-G/K gene. J. Biol Chem 1993; 268:18912-18916

[65] Nakamoto H, Hasegawa M Targeted inactivation of the gene psaK encoding a subunit of photosystem I from the cyanobacterium Synechocystis sp. PCC 6803. Plant Cell Physiol. 1999; 40:9-16. 
[66] Morosinotto T, Breton J, Bassi R, Croce R The nature of chlorophyll ligand in Lhca proteins determines the far red fluorescence emission typical of photosystem I. J. Biol. Chem. 2003; 278(49):49223-49229

[67] Koziol A.G, Borza T, Ishida K, Keeling P, Lee L.W, Durnford D.G, Tracing the evolution of the light-harvesting antennae in chlorophyll a/b-containing organisms. Plant Physiol. 2007; 143:1802-1816.

[68] Zhang H, Goodman H.M, Jansson S, Antisense inhibition of the Photosystem I Antenna protein Lhca4 in Arabidopsis thaliana. Plant Physiol. 1997; 115(4):1525-31

[69] Haworth P, Watson J.L, Arntzen C.J, The detecting isolation and characterization of a light-harvesting complex which is specifically associated with Photosystem I. Biochim. Biophys. Acta 1983; 721:151-158

[70] Lam E, Ortiz W, Malkin R, Chlorophyll a/b proteins of photosystem I. FEBS Lett $1984 ; 168: 10-14$

[71] Gobets B, van Grondelle R, Energy transfer and trapping in Photosystem I. Biochim. Biophys. Acta 2001;1507:80-99

[72] Neilson J.A, Durnford D.G Structural and functional diversification of the light harvesting complex in photosynthetic eukaryotes. Photosyth. Res. 2010;106:54-71

[73] Ihalainen J. A, Jensen P. E, Haldrup A, van Stokkum I. H. M, van Grondelle R, Scheller H. V., Dekker J. P Pigment organization and energy transfer dynamics in isolated photosystem I (PSI) complexes from Arabidopsis thaliana depleted of the PSI-G, PSIK, PSI-L, or PSI-N subunit. Biophys. J. 2002; 83, 2190-2201

[74] Mullet J.E, Burke J.J, Arntzen C.J, Chlorophyll proteins of Photosystem I. Plant Physiol. $1980 ; 65: 814-822$.

[75] Mozzo M, Morosinotto T, Bassi R, Croce R Probing the structure of Lhca3 by mutation analysis, Biochim. Biophys. Acta 2006;1757:1607-1613.

[76] Lelong C, Setif P, Lagoutte B, Bottin H Identification of the amino acids involved in the functional interaction between photosystem I and ferredoxin from Syneckocystis sp. PCC 6803 by chemical cross-linking. J Biol Chem 1994;269 10034-10039

[77] Xu Q, Jung Y.S, Chitnis V.P, Guikema J.A, Golbeck J.H, Chitnis P.R Mutational analysis of photosystem I polypeptides in Synechocystis sp. PCC 6803. Subunit requirements for reduction of NADP+ mediated by ferredoxin and flavodoxin. J Biol Chem. 1994; 269(34):21512-21518.

[78] Varotto C, Pesaresi P, Jahns P, Lessnick A, Tizzano M, Schiavon F, Salamini F, Leister D Single and double knock-outs of the genes for photosystem I subunits $G, K$ and $H$ of Arabidopsis. Effects on photosystem I composition, photosynthetic electron flow and state transtions. 2002; Plant Physiol. 129:616-624 
[79] Lunde C, Jensen P.E, Haldrup A, Knoetzel J Scheller H.V The PS I-H subunit of Photosystem I is essential for state transitions in plant photosynthesis. Nature 2000; 408: 613-615

[80] Naver H., Haldrup A, Scheller H.V Cosuppression of photosystem I subunit PSI-H in Arabidopsis thaliana. Efficient electron transfer and stability of photosystem I is dependent upon the PSI-H subunit, J. Biol. Chem. 1999; 274:10784-10789

[81] Haldrup A, Simpson D.J., Scheller H.V Down-regulation of the PSI-F subunit of photosystem I in Arabidopsis thaliana. The PSI-F subunit is essential for photoautotrophic growth and antenna function, J. Biol. Chem. 2000;275:31211-31218.

[82] Wöstemeyer A.; Oelmüller R The promoter of the spinach PSaF gene fort he subunit III of the photosystem I reaction center directs $\beta$-glucuronisade gene expression in transgenic tobacco roots, implication of the involment of phospholipases and protein kinase C in PsaF gene expression J.Plant Physiol. 2003;160: 503-508.

[83] Ihalainen J. A, Jensen P. E, Haldrup A, van Stokkum, I. H. M., van Grondelle R., Scheller, H. V, Dekker, J. P Pigment organization and energy transfer dynamics in isolated photosystem I (PSI) complexes from Arabidopsis thaliana depleted of the PSI-G, PSI-K, PSI-L, or PSI-N subunit. Biophys. J. 2002; 83:2190-2201

[84] Oh-oka H, Takahaski Y, Kuriyama K, Saeki K, Matsubara H The protein responsible for center $\mathrm{A} / \mathrm{B}$ in spinach photosystem I: isolation with iron-sulfur cluster(s) and complete sequence analysis. J. Biochem (Tokyo) 1988;103:962-68

[85] Okkels J.S, Scheller H.V, Jepsen L.B, Møller B.L A cDNA clone encoding the precursor for a 10.2 kDa photosystem I polypeptide of barley. FEBS Lett 1989; 250: 575-579

[86] Okkels J.S, Nielsen V.S, Scheller H.V, Møller B.L A cDNA clone from barley encoding the precursor from the photosystem I polypeptide PSI-G: sequence similarity to PSI-K. Plant Mol Biol 1992;18: 989-994

[87] Kusnetsov V, Bale C, Lübberstedt T, Hermann R.G, Oelmüller R Evidence that the plastid signal and light operate via the same cis-acting elements in the promoters of nuclear genes for plastid proteins. Mol. Gen. Genet. 1996; 252:631-639

[88] Ikeuchi M, Nyhus K.J, Inoue Y, Pakrasi H.B Identities of four low-molecular-mass subunits of the photosystem I complex from Anabaena variabilis ATCC 29413 evidence for the presence of the psaI gene product in cyanobacterial complex. FEBS 1991; 287(1):5-9

[89] Chen S.C.G, Cheng M.C, Chen J, Hwang L.Y Organization of the rice chloroplast psaA-psaB-rps14 gene and the presence heterogeneity in this gene cluster. Plant Sci $1990 ; 68: 213-221$

[90] Chen S.C.G, Cheng M.C, Chung K.R, Yu N.J, Chen M.C Expression of the rice chloroplast of the rice chloroplast psaA-psaB-rps14 gene cluster. Plant Sci 1992;81:93-102 
[91] Cheng M.C, Wu S.P, Chen L.F.O, Chen S.C.G Identification and prufication of a spinach chloroplast DNA-binding protein that interacts specifically with the plastid psaA-psaB-rps14 promoter region Planta 1997;203:373-380

[92] Kusnetsov V, Bale C, Lubberstedt T, Sapory S, Hermann R.G, Oelmüller R Evindence that the plastid signal and light operade via the same cis-acting elements in the promoters of nuclear genes for plastid proteins. Mol. Gen. Genetic 2006;252:631-639

[93] Kropat J, Oster U, Rudiger W, Beck C.I Chlorophyll precursors are signals of chloroplast origin involved in light induction of nuclear heat-schock genes Proc. Natl. Acad. Sci. USA 1997;94:14168-14172

[94] Mochizuki N, Brusslan J.A, Larkin R, Nagatani A, Chory J Arabidopsis genomes uncoupled 5 (GUN5) mutant reveals the involvement of Mg-chelatase H subunit in plastid-to-nucleus signal transduction. Proc. Natl. Acad. Sci. USA 2001; 98:2053-2058

[95] Kusnetsov V, Oelmüller R, Sarwat M.I, Porfirova S.A, Cherepneva G.M, Hormann R.G, Kulaeva O.N Cytokinins, abscisic acid and light affect on accumulation of chloroplast proteins in Lupinus luteus cothyledons without notable effect on steady state mRNA levels. Planta 1994;194:318-327

[96] Yamamoto Y.M, Tsuji H, Obokata J 5'-leader of a photosystem I gene in Nicotiana slyvestris, psaDb, contains a translational enhancer. J. Biol. Chem. 1995; 270(21): $12466-12470$

[97] Kusnetsov V, Landsberger M, Neuer J, Oelmüller R, The assembly of the CAAT-box binding complex at the AtpC promoter is requlated by light, cytokinin and the stage of the plastids. J. Biol. Chem. 1999; 274:36009-36014

[98] Flieger K, Tyagi A, Sopary S, Cseplö A, Herrmann RG, Oelmüller R A 42 bp promoter fragment of the gene for subunit III of photosystem I (psaI) is crucial for its activity Plant J. 1993;4:9-17

[99] Oelmüller R, Lübberstedt T, Bolle C, Scpory S, Tyagy A, Cseplö A, Fliegr K, Herrmann R.G 1992. Promoter architecture of nuclear genes for thylakoid membrane proteins from spinach. In: Murata(ed) Research in Photosysthesis Vol III. Kluwer Acad. Publishers, Dordirecht pp. 219-224

[100] Gilmartin P.M, Memelink S, Hiratsuka K, Kay S.A, Chua N.HCharacterization of gene encoding a DNA binding protein with specificity for a light-responsive element. Plant Cell 1992;4:839-849

[101] Ihnatowicz A, Pesaresi P, Varotto C, Richly S, Scheider A, Jahns P, Salamini F, Leister D Mutants of photosystem I subunit D of Arabidopsis thaliana:effects on photosysthesis, photosystem I stability and expression of nuclear genes for chloroplast functions. Plant J. 2004;37:839-852. 
[102] Allakhverdiev S. I, Sakamoto A, Nishiyama Y, Inaba M, Murata N Ionic and osmotic effects of NaCl-induced inactivation of photosystem I and II in Synechococcus sp. Plant Physiol 2000;123:1047-1056.

[103] Zhang T, Gong H, Wen X., Lu C Salt stress a decrase in excitation energy transfer from phycobilisomes to photosystem II but an increase to photosystem I in the cyanobacterium Spirulina platensis. J Plant Physiol. 2010;167:951-958.

[104] Jones L.W, Kok B Photoinhibition of chloroplast reaction I. Kinetics and action spectra. Plant Physiol 1966; 41:1037-1043

[105] Kok B On the inhibition of photosynthesis by intense light. Biochm. Biophys. Acta $1956 ; 21: 234-244$

[106] Sato K Mechanism of photoinactivation in photosynthetic systems. I. The dark reaction in photoinactivation. Plant Cell Physiol. 1970; 11:15-27

[107] Sato K Mechanism of photoinactivation in photosynthetic systems. II. The occurrence and properties of two different types of photoinactivation. Plant Cell Physiol. 1970; $11: 29-38$

[108] Inoue K, Sakurai H, Hiyama T Photoinactivation site of photosystem I in isolated chloroplasts. Plant Cell Physiol. 1986; 27:961-968

[109] Inoue K, Fujii T, Yakoyama E, Matsuura K, Hiyama T, Sakurai H The photoinhibition site of photosystem I in isolated chloroplasts under extremely reducing conditions. Plant Cell Physiol. 1989; 30:65-71

[110] Sonoike K Photoinhibition of photosystem I. Physiol. Plantarum 2011;142:56-64

[111] Teroshima I, Funayama S, Sonoike K The site of photoinhibition in leaves of Cucumis sativus L. at low temperature is photosystem I, not photosystem II. Planta 1994;193:300-306

[112] Sonoike K., Terashima Mechanism of the photosystem I photoinhibition in leaves of Cucumis sativus L. Planta 1994;194: 287-293

[113] Asada K. 1999 Radical production and scavenging in the chloroplasts. In: Baker NR(ed) photosynthesis and the environment Kluwer Academic Publishers, Dordrecht, pp 123-150

[114] Ivanov A.G, Morgan R.M, Gray G.R, Velitchkova M.Y, Huner N.P.A Temperature/ light dependent development of selective resistance to photoinhibition of photosystem I. FEBS Lett. 1998;430:288-292

[115] Prasil O, Adir N, Ohad I 1992 Dynamics of photosystem I: Mechanism of photoinhibiton and recovery process. In Topics in Photosynthesis: the Photosystem Structure, Function and Molecular Biology, Edited by Barber J. pp. 295-348. Elsevier, Amsterdam 
[116] Aro E.M, Virgin I, Anderson B, Photoinhibition of photosystem II. Inactivation, protein damage and turnover. Biochim. Biophys. Acta 1993;1143:113-134

[117] Andersson B, Barber J 1996. Mechanisms of photodamage and protein degradation during photoinhibition of photosystem II. In Photosynthesis and Enviroment. Edited by Baker NR, pp. 101-121. Kluwer Academic, Dordrecht

[118] Teicher H.B., Møller B.L, Scheller H.V photoinhibiton of photosystem I in fieldgrown barley (Hordeum vulgare L.): Induction, recovery and acclimation Photosynth. Res. 2000 64:53-61

[119] Kudah H, Sonoike K Irreversible damage to photosystem I by chilling in the light: cause of the degradation of chlorophyll after returaing to normal growth temperature. Planta 2002;215:541-548

[120] Zhang S, Scheller H.V Photoinhibition of photosystem I at chilling temperature and subsequent recovery in Arabidopsis thalliana Plant Cell. Physiol. 2004;45(11):1595-1602

[121] Murchie E. H, Hubbart S, Chen Y, Peng S, Horton P Acclimation of Rice Photosynthesis to Irradiance under Field Conditions. Plant Physiol. 2005;130, 1999-2010.

[122] Sonoike K Selective Photoinhibition of photosystem I In isolated thylakoid membrane from cucumber and spinach. Plant Cell Physiol. 1995; 36, 825-830.

[123] Ogawa K, Kanematsu S, Takabe K. Asada K. Attachment of Cu/Zn-superoxide dismutase to thylakoid membranes at the site of superoxide generation (PS1) in spinach chloroplasts: detection by immuno-gold labeling after rapid freezing and substitution method. Plant cell Physiol. 1995;36, 565-573.

[124] Tjus S. E, Møller B. L, Scheller H. V Photoinhibition of photosystem I damages both reaction center proteins PSI-A and PSI-B and acceptor-side located small photosystem I. Photosynth. Res. 1999; 60, 75-86

[125] Jiao D, Emmanuel H Guikema, J. A High light stress inducing photoinhibition and protein degradation of photosystem I in Brassica rapa. Plant Sci. 2004;167, 733-741.

[126] Lu C, Vonshak A, Characterization of PSII photochemistry in salt-adapted cells of cyanobacterium Spirulina platensis. New Phytol. 1999;141:231-239.

[127] Lu C, Vonshak A, Effects of salinity stres on photosystem II function in cyanobacterial Spirulina platensis cells. Physiol. Plant 2002;114:405-413.

[128] Gilmour D.J, Hipkins M.F, Boney A.D The effects of salt stres on the primary processes of photosynthesis in Dunalielia tertioleca. Plant Sci. Lett. 1982;26:325-330.

[129] Joset F, Jeanjean R, Hagemann M Dynamics of the response of cyanobacteria to salt stres:deciphering the molecular events. Physiol Plant. 1996; 738-744. 
[130] Sudhir P. R, Pogoryelov D, Kovács L, garab G, Murthy S.D.S The effects of salt stres on photosynthetic electron transport and thylakoid memebrane proteins in the cyanobacterium Spirulina platensis. J Biochem Mol Biol. 2005;38:481-485.

[131] Hibino T, Lee B.H, Rai A.K, Ishikawa H, Kojima H, Tawada H, Shyimoyama H, Takebe T Salt enhances photosystem I content and cyclic electron flow via NAD $(\mathrm{P}) \mathrm{H}$ dehydrogenase in the halotolerant cyanobacterium Aphanothece halophytica. Aust J Plant Physiol. 1996;23:321-330

[132] Jeanjean R, Matthijs H.C.P, Onana B, Havaux M, Joset F Exposure of the cyanobacterium Synechocystis PCC 6803 to salt stres induces concerted changes in respiration and photosynthesis. Plant Cell Physiol. 1993;34:1073-1079.

[133] Neelam A, Rai L. C Differantial responses of three cyanobacteria to UV-B and Cd, Microbiol. Biotechnol 2003;13:544-551.

[134] Zhou W, Juneau P, Qiu B Growth and photosynthetic responses of the bloom-forming cyanobacterium Microcystis aeruginosa to elevated lecels of cadmium, Chemosphere 2006;65:1738-1746.

[135] Ivanov A. G, Morgan R. M, Gray G.R, Velitchkova M. Y,. Huner N.P.A Temperature/ light dependent development of selective resistance to photoinhibition of photosystem I, FEBS Lett 1998;430:288-292.

[136] Jin S.H, Li X.Q, Hu J.Y, Wang J.G Cyclic electron flow around photosystem I is required for adaptation to high temperature in a subtropical forest tree, Ficus concinna. J Zhejiang Univ Sci B. 2009;10(10):784-790.

[137] Howitt C. A, Cooley J. W, Wiskich J. T, Vermaas W. F A strain of Synechocystis sp. PCC 6803 without photosynthetic oxygen evolution and respiratory oxygen consumption: implications for the study of cyclic photosynthetic electron transport, Planta 2001;214:46-56.

[138] Jeanjean R, Bedu S, Havaux M, Matthijs H. C. P, Joset F Salt-induced photosystem I cyclic electron transfer restores growth on low inorganic carbon in a type I NAD $(\mathrm{P}) \mathrm{H}$ dehydrogenase deficient mutant of Synechocystis PCC6803, FEMS Microbiol. Lett 1998;167:131-137.

[139] Tanaka Y, Katada S, Ishikawa H, Ogawa T, Takabe T Electron flow front NAD $(\mathrm{P}) \mathrm{H}$ dehydrogenase to photosystem I is required for adaptaion to salt shock in the Cyanobacterium Synechocystis sp. PCC 6803, Plant Cell Physiol 1997;38:1311-1318. 